\title{
RECICLAJE, APRENDIZAJE COLABORATIVO Y USO DE LAS TIC: "PROYECTO CARTONERO"
}

\author{
Recycling and Collaborative Learning and Use of ICT: "Cartonero Project"
}

\author{
Mónica Milagros Lanchipa Bergamini ${ }^{1}$
}

\begin{abstract}
RESUMEN
“Construyendo libros cartoneros, protegemos el medio ambiente y fomentamos prácticas sociales de lectura y escritura, a través de las TIC", es un proyecto dirigido a alumnas de quinto y sexto grado de la I.E. Santísima Niña María de Tacna-Perú y participan en su ejecución: estudiantes, profesores, padres de familia, autoridades, etc. La población beneficiaria asciende a 300 personas cada año. El problema que ayuda a mitigar el proyecto, es el deterioro del medio ambiente, reutilizando el cartón obtenido de los mercadillos de Tacna, para confeccionar libros creativos, y hacer evidente que el reciclaje es posible y necesario. Posteriormente se afianzó con los libritos cartoneros el proceso de lectura y escritura y se fomentaron las prácticas sociales, usando las Tecnologías de Información y Comunicaciones (TIC), para fortalecer la competencia lectora, así como despertar la conciencia ambiental. Para este fin, se diseñó un ambiente de aprendizaje presencial mediado por las TIC, en el cual se propuso una serie de estrategias y actividades que fueron compartidas en la web a través del blog y Facebook del proyecto. Esta práctica fue implementada en el 2009 y mejorada a través de los años, siendo premiada en varias oportunidades.
\end{abstract}

Palabras clave: Medio ambiente, reciclaje, aprendizaje colaborativo, prácticas sociales, Tecnologías de Información y Comunicaciones.

\section{ABSTRACT}

"Building cardboard books, we protect the environment and foster social practices of reading and writing, through ICT" is a project aimed at students in fifth and sixth grade in Holy Child Mary School of Tacna-Peru and students, teachers, parents, authorities, etc. participate in its implementation. The target population is 300 people every year. The problem that helps mitigate the Project is the environmental deterioration. By reusing cardboard obtained from the markets of Tacna, to make creative books, and make clear that recycling is possible and necessary. After, with the use of cardboard booklets the process of reading and writing and social practices were promoted, using Information and Communication Technologies (ICT) to strengthen reading skills, and raise environmental awareness. To get this aim, a face learning environment mediated by ICT was designed, in which a number of strategies and activities that were shared on the web through blog and Facebook were proposed. This practice was implemented in 2009 and improved over the years, being awarded several times.

Keywords: environment, recycling, collaborative learning, social practices, Information Technology and Communications.

\footnotetext{
${ }^{1}$ Licenciada en Administración. Institución Educativa Santísima Niña María. Tacna - Perú. Email:lanbermo8@hotmail.com
} 


\section{INTRODUCCIÓN}

En América Latina el problema de la basura se va incrementando con el paso del tiempo, ya que a medida que la población ha ido creciendo también lo ha hecho la cantidad de desechos que se produce. En el año de 1960, la Organización Panamericana de la Salud (OPS), adopta la primera resolución sobre desechos sólidos e invita a los ministerios de Salud de América a que adopten estas medidas.

La basura se convierte en una fuente de expansión de enfermedades y de contaminación del agua; además, durante la estación seca, a menudo entra en combustión espontánea, contaminando el aire y causando serios problemas a la población que vive en las cercanías. No hay ciudad o pueblo en que la recogida de desechos llegue a todos los rincones $y$, en algunos casos, ni siquiera a la mayoría de las viviendas. Como resultado, la basura se vierte en las calles, en los ríos, etc. El problema afecta a todo el país y está empeorando rápidamente, debido a la alta presión demográfica y a la falta de aplicación de medidas efectivas.

Los desechos sólidos comprenden material inorgánico: papel, cartón, vidrio, plástico, tela, latas de aluminio y otros metales.

En el Perú el problema de la basura se va incrementando con el paso del tiempo, con el crecimiento de la población también crece la cantidad de desechos.

El departamento de Tacna no es ajeno a estos hechos ya que se encuentra en una zona altamente comercial y turística, a esto hay que agregar que es uno de los departamentos peruanos con mayor crecimiento poblacional actualmente, es por ello que resultó imprescindible la necesidad de formular una alternativa de solución. De acuerdo a nuestras investigaciones, evidenciamos que en los mercadillos de Tacna existe una gran cantidad de cartón desechado como basura al final del día. Por lo cual decidimos fijar nuestra atención en ese cartón, investigamos las formas de reciclarlo y nos propusimos tratar de ayudar con un granito de arena a este gran problema de la contaminación ambiental que nos afecta a todos, utilizando todos los medios informáticos para divulgar nuestra experiencia y colaborar con el cuidado del planeta.

\section{Definición de términos básicos:}

- Contaminación:Se entiende por contaminación la adición de cualquier sustancia al ambiente en suficientes cantidades, que causen efectos mensurables o medibles sobre los seres humanos, los animales, la vegetación o los materiales y que se presenten en cantidades que sobrepasen los niveles normales de los que se encuentran en la naturaleza. Es el deterioro, alteración, contagio, desequilibrio y toda otra acción que afecte negativamente el equilibrio natural o el estado de sanidad de organismos vivientes y no vivientes. La contaminación y la polución son sinónimos. Por lo anterior el hombre va camino a envenenar toda la tierra, sin dejar ningún posible refugio para una reserva de vida y salud.

- Cartón: El cartón es un material constituido por una plancha gruesa de pasta de papel endurecida o por varias hojas de papel superpuestas y adheridas unas a otras. El cartón es una lámina gruesa y dura de pasta de papel endurecida por compresión.

- Problema Ambiental: Situación o configuración de factores que amenaza el bienestar humano o la integridad del ecosistema, y que es percibida como tal por la sociedad o una parte de ella. 
- Protección del Medio Ambiente: El conjunto de políticas, planes, programas, normas y acciones destinadas a mejorar el medio ambiente y a prevenir y controlar su deterioro.

- Medio Ambiente: Es todo aquello que nos rodea y que debemos cuidar para mantener limpia la ciudad, colegio, hogar. Es el conjunto de elementos abióticos (energía solar, suelo, agua y aire), bióticos (organismos vivos) que integran la delgada capa de la tierra llamada biosfera, sustento y hogar de los seres vivos.

- Biodiversidad: La variabilidad entre los organismos vivos, que forman parte de todos los ecosistemas terrestres y acuáticos. Incluye la diversidad dentro de una misma especie, entre especies y entre ecosistemas.

- Calidad de Vida: Concepto que integra el bienestar físico, mental, ambiental y social como es percibido por cada individuo y cada grupo. Depende también de las características del medio ambiente en que el proceso tiene lugar (urbano, rural).

- Suelo: Capa o conjunto de capas del terreno procedentes de la transformación de una roca madre subyacente o preexistente, como consecuencia de la actuación del complejo de factores en que figura e interviene la vida.

- Reciclaje: Volver a utilizar. Es la obtención de materias primas a partir de la reutilización, sin tocar los recursos naturales introduciéndolo nuevamente al circuito de uso. Proceso mediante el cual se vuelven a utilizar las materias de desecho ya usadas, las cuales son transformadas en nuevos productos. En nuestro país se está comenzando con algún éxito en el reciclado de papel, cartón, latas y envases de vidrios. Se está experimentando con los PET (envase de plásticos de gaseosas, aceites, vinos, etc.). Se reciclan también residuos domésticos orgánicos, elementos esenciales para fabricar compost.

- Contaminante:Sustancia o compuesto que afecta negativamente al ecosistema. Se reconocen dos tipos de contaminantes: Los no biodegradables, llamados así porque no se descomponen o lo hacen muy lentamente, tales como recipientes de vidrio, plástico, latas, el D.D.T., Malatión, y otros; los contaminantes biodegradables, que se descomponen con relativa rapidez o facilidad: Aguas negras (aguas cloacales), algunos tipos de detergentes y los restos orgánicos.

- TIC: Las Tecnologías de la Información y la Comunicación son un conjunto de servicios, redes, software y aparatos que tienen como fin la mejora de la calidad de vida de las personas dentro de un entorno, y que se integran a un sistema de información interconectado y complementario. También las TIC son el conjunto de tecnologías desarrolladas para gestionar información y enviarla de un lugar a otro. Están presentes en nuestras vidas y la han transformado.

- Aprendizaje Colaborativo: En su sentido básico, aprendizaje colaborativo (AC) se refiere a la actividad de pequeños grupos desarrollada en el salón de clase. Aunque el AC es más que el simple trabajo en equipo por parte de los estudiantes, la idea que lo sustenta es sencilla: los alumnos forman "pequeños equipos" después de haber recibido instrucciones del profesor. Dentro de cada equipo, los estudiantes intercambian información y trabajan en una tarea hasta que todos sus miembros la han entendido y terminado, aprendiendo a través de la colaboración.

- Prácticas sociales de lectura y escritura: La lectura y la escritura son dos pro- 
cesos fundamentales en la historia del hombre, en el desarrollo de la humanidad y en la adquisición y estructura de nuevos conocimientos. La escuela busca desarrollar las prácticas sociales del lenguaje para que así como se realizan en la escuela las puedan aplicar en lo cotidiano. Las prácticas sociales del lenguaje están orientadas a la comunicación de los hablantes en situaciones particulares de su cultura.

\section{Objetivo general:}

Dar a conocer a la población una forma fácil, económica y divertida, de elaborar un libro, fomentar la lectura y escritura a través de las TIC; y a su vez contribuir con la protección del medio ambiente.

\section{Objetivos específicos:}

- Promover la lectura y escritura con prácticas sociales en la institución educativa.

- Utilizar las TIC en los procesos creativos, en las prácticas sociales de lectura y escritura, y en la difusión del proyecto.

- Proteger el medio ambiente y crear conciencia ecológica, reciclando el cartón, así como mejorando las actitudes y habilidades de las estudiantes en materia de educación ambiental.

- Estimular la creatividad y la expresión artística, al confeccionar, diseñar y escribir el libro cartonero.

- Promover la responsabilidad social y ambiental de los docentes y estudiantes mediante la participación en la mejora de su entorno. Y fomentar la participación activa de sus familias y la comunidad en general, para mejorar su desempeño ambiental con eficiencia.

\section{Relevancia del proyecto}

- Se inspira en la misión, visión y valores de la I.E. Santísima Niña María de Tacna.

- Facilita el desarrollo de actividades de aprendizaje creativas en las diferentes áreas.

- Responde a una necesidad identificada (recojo de basura).

- Es innovadora (imaginación y creatividad).

- Es efectiva.

- Es flexible.

- Desarrolla amplia participación de los miembros de la familia escolar.

- Cuenta con los recursos necesarios para implementarse en cualquier contexto.

- Permite la retroalimentación y la mejora continua.

\section{Coherencia con los lineamientos de política educativa nacional, regional y/o local}

El proyecto de innovación: "Construyendo libros cartoneros protegemos el medio ambiente y fomentamos prácticas sociales de lectura y escritura, a través de las TIC", se encuentra en concordancia con las prioridades y planes de desarrollo local, regional y nacional. La Municipalidad Provincial de Tacna, el Gobierno Regional y el Gobierno Central, coinciden y tienen como objetivo la protección y conservación del medio ambiente y los recursos naturales regulando las acciones del hombre con relación a la naturaleza y promoviendo el desarrollo sostenible con la finalidad de mejorar la calidad de vida de la población.

El Perú del siglo XXI se nos presenta como un país rico en diversidad, en constante crecimiento económico y habitado además por gente tenaz, perseverante y creativa. En este momento de nuestra historia, el país necesita más que 
nunca una educación que forme a las personas capaces de convertir ese potencial en oportunidades de desarrollo y progreso para todos. Personas que, desde la diversidad que nos caracteriza, hagan suyo el compromiso de fortalecer nuestra identidad común, de hacer de la democracia una manera deseable de vivir y de gobernarnos a nosotros mismos. Este proyecto educativo está relacionado con la finalidad de la educación en el Perú (Ley General de Educación), que es formar personas capaces de lograr su propia realización. Para ello, deben desarrollar competencias en cuatro ámbitos: la afirmación de su identidad y autoestima, el ejercicio de su ciudadanía en armonía con su entorno social y ambiental, la vinculación al mundo del trabajo y los retos en el mundo del conocimiento. He aquí nuestro aporte en lo que se refiere a mejorar la conciencia ambiental, promover la lectura y escritura y estimular la creatividad.

Los ocho aprendizajes fundamentales que propone la tercera versión del marco curricular como horizonte común para todo el país, no son disonantes, con nuestro proyecto cartonero. Específicamente se relacionan con las áreas de Comunicación eficaz, de forma escrita a través de las creaciones plasmadas en los libros cartoneros y la comunicación oral al compartir las creaciones con sus pares; el cuidado de la naturaleza, promoviendo la protección del medio ambiente y generando una conciencia ecológica en las niñas, además de aplicar conocimientos científicos y tecnológicos al compartir sus aprendizajes en los blog y redes sociales del proyecto; Arte y creatividad, al diseñar las tapas de los libros cartoneros con originalidad; Personal Social, actuando con plena conciencia de sus derechos y deberes ambientales; es decir, el proyecto está alineado a los consensos y prioridades que son promo- vidos por los lineamientos de política educativa nacional, regional y/o local.

\section{CONSTRUYENDO LIBROS CARTONEROS}

\section{Descripción}

El proyecto se denomina "Construyendo libros cartoneros, protegemos el medio ambiente y fomentamos prácticas sociales de lectura y escritura a través de las TIC", más conocido durante todos estos años (desde el 2009 en adelante) como "Niñita Cartonera" en honor a la patrona de nuestra institución educativa "Santísima Niña María" de Tacna. El proyecto está inmerso en el Plan Anual de Trabajo (PAT) de la I.E. Santísima Niña María.

Esta experiencia está dirigida al V ciclo (quinto y sexto grado), y participan en su ejecución alumnas, profesores, padres de familia, personajes de la comunidad, autoridades, etc. La población beneficiaria asciende a 300 personas. Además se encuentra en concordancia con las prioridades y planes de desarrollo local, regional y nacional. Coincide con el objetivo de protección y conservación del medio ambiente y los recursos naturales. El problema que se trata de resolver o mitigar con el presente proyecto cartonero, es el deterioro del medio ambiente, y al desarrollar la experiencia se ayudará a preservarlo y a frenar la contaminación. Por eso reutilizamos el cartón, obtenido de los mercadillos de Tacna, para confeccionar libros creativos y divertidos, y hacer evidente que el reciclaje es posible y necesario. Otro de los objetivos que pretendo lograr con la experiencia, es mejorar el proceso de lectura y escritura, para lograr la competencia lectora, tan importante dentro del Proyecto Educativo Nacional, así como despertar la capacidad creadora en las alumnas con el fin de 
prepararlas para un mundo actual y competitivo.

Las estrategias a utilizar son variadas, los instrumentos innovadores y creativos, la metodología activa; todo ello dirigido a lograr captar la atención de la alumna para que nadie se quede atrás. Nuestro compromiso como docentes es mejorar los aprendizajes a través del proyecto cartonero el cual viene ejecutándose en la institución educativa Santísima Niña María desde el 2009.

\section{Propuesta Pedagógica}

\section{Estrategias de enseñanza aprendizaje:}

Se proponen dos niveles de estrategias: individuales y grupales, que contribuyan en la búsqueda de informaciones en la Web y otras fuentes, la interacción tutora-alumna y alumna-alumna, la retroalimentación y el aprendizaje colaborativo a través de las TIC. Se han diseñado estrategias de enseñanza referidas a procedimientos e instrucciones para ayudar a las alumnas a integrar nuevos conocimientos y motivarlas para que desarrollen estrategias de aprendizaje o procedimientos que emplearían para aprender significativamente. Se asume como estrategias de enseñanza, organizar la información que se ha de aprender. En este caso, los contenidos están divididos en una estructura secuencial y en un lenguaje sencillo con el "fin de facilitar el aprendizaje significativo de las alumnas". Otra estrategia, es alentar el aprendizaje significativo, establecer expectativas y orientar la atención motivando la participación de estas alumnas para las cuales se ha diseñado este proyecto, donde la comunicación debe ser permanente. Igualmente, para promover el enlace entre el conocimiento y la información que se ha de aprender. La puesta en práctica de este proyecto, ayudará a desarrollar habilidades analíticas que buscan relacionar mediante el análisis ideas y conceptos; la búsqueda de información a partir de las consignas de las clases; inventivas y creativas, desarrolladas en el trabajo colaborativo mediante la lluvia de ideas y desarrollo de pensamiento lateral en la discusión de los temas del sistema de conocimiento; de comunicación desarrollada en las exposiciones de grupo, y de asimilación y retención de la información.

Las estrategias pedagógicas que más contribuyeron con los logros de los aprendizajes, fueron las que se utiliza para estimular desde el aula de clase, el uso de las habilidades tecnológicas, pensamiento y creatividad en las alumnas. La propuesta buscó alentar a las alumnas para que en su quehacer diario, empleen dichas propuestas de lectura y escritura creativa, apoyadas por las TIC. La segunda propuesta, fue incentivar la conciencia ambiental, planteando el empleo del cartón reciclado para construir libros creativos y divertidos, como estrategia pedagógica que permita estimular el desarrollo de las habilidades del pensamiento creativo en las alumnas. Y la tercera propuesta está referida a expresarse con los lenguajes del arte y apreciarlo en sus diferentes manifestaciones, al diseñar y pintar las tapas de sus libros de su propia autoría.

\section{Actividades que se desarrollaron:}

Los principales logros se han efectivizado a través de las siguientes actividades:

Logro 1: Mejora la lectura y escritura en las alumnas del plantel, apoyadas por las TIC.

- Construcción del librito cartonero.

- Aplicación del librito cartonero en actividades de aprendizaje de las diferentes áreas.

- Uso de las TIC como apoyo en los procesos creativos, en las prácticas 
sociales de lectura y escritura, y en la difusión del proyecto.

- Formación de grupos motivadores.

- Grupos motivadores en acción, desarrollando prácticas sociales de lectura en grados menores.

- Talleres de fortalecimiento de la comprensión de lectura.

- Concurso de comprensión de lectura.

\section{Logro 2: Protege el medio ambiente}

- Charlas con especialistas de la Municipalidad Provincial de Tacna acerca del recojo de la basura.

- Talleres de recojo de cartón de los mercadillos de Tacna.

- Elaboración de libritos cartoneros dedicados a diferentes temas: medio ambiente, valoración de la mujer, la familia, motivos peruanos, aniversario $\mathrm{N}^{\circ} 50$ de Mafalda, etc.

- Confección de trajes e instrumentos reciclados para presentación en el "Encuentro Binacional de Cuidado del Medio Ambiente".

- Concurso al mejor equipo de alumnas que trabajan incentivando la conciencia ambiental y la protección del medio ambiente, dentro del colegio y fuera de ella.

- Charlas sobre la importancia de cuidar el medio ambiente.

- Construcción de otros materiales en base de cartón reciclado.

- Colaboración en laimplementación de bibliotecas ambientales.

- Alianza con otras instituciones interesadas en el cuidado del medio ambiente: D'Cada Ciudadano, Universidad Nacional Jorge Basadre Grohmann de Tacna, Autoridad Nacional del Agua (ANA), Consulado General de Chile, Cáritas (Fronteras Unidas por un Ambiente Sano), etc.
Logro 3: Fomenta la creatividad y expresión artística

- Talleres de arte y creatividad.

- Charla a padres de familia con el objetivo de enseñarles a incentivar la creatividad en sus niñas.

- Construcción del librito cartonero de los padres de familia.

- Talleres de autoestima con participación de padres e hijas (participación obligatoria).

- Elaboración de cajas artísticas denominadas: "Mis recuerdos del nivel primario" utilizando cajas de pizza recicladas.

- Concurso a la mejor experiencia creativa de grupos motivadores en el fomento de las prácticas sociales de lectura y escritura, en las alumnas de grados menores.

- Campañas de sensibilización a los padres y madres de familia acerca de la importancia de leer creativamente en familia.

- Organización y participación en las Ferias Pedagógicas y Festival de la Lectura y la Creatividad de la I.E. Santísima Niña María.

- Organización y participación en el "Festival de las Artes en Cartón".

- Muestra anual de inventos, utilizando "chatarra tecnológica".

\section{Recursos que se utilizaron}

Materiales:

a) Materiales para elaboración de libros cartoneros: Cajas de cartón, témperas, plumones, colores, pinceles, hojas de papel bulki, goma, grapas, cuchilla, tijera, regla, etc.

b) Materiales para exposición del proyecto: libritos cartoneros, otros objetos realizados en cartón, carteles de cartón, telas de colores, etc.

c) Materiales impresos de enseñanza: libros de texto, enciclopedias, fichas de actividades, cómics, diccionarios, 
cuentos, obras, etc.

*Se utilizaron para la etapa de investigación, previos a la creación de diversos textos en el librito cartonero, con la finalidad de promover la creatividad y estimular la imaginación del educando.

\section{Medios:}

a) Medios Visuales: Carteles, murales, periódicos, revistas, rotafolios, trípticos, diapositivas, pizarrón, etc.

b) Medios Auditivos: Palabra hablada (exposición-diálogo), radio.

c) Medios Audiovisuales: Video, televisión, informáticos (Presentaciones didácticas en la web, herramientas multimedia, video interactivo), telemáticos (Internet, correo electrónico, ambiente virtual de aprendizaje).

*Se utilizaron en las diferentes etapas de implementación de la buena práctica, para incentivar al alumnado de la I.E. Santísima Niña María, a los padres de familia y a la comunidad en general, a participar en dicho proyecto $\mathrm{y}$ para difundir los logros obtenidos.

d) Medio (software): plataformas educativas, aplicaciones multimedia, blogs, wikis, videos, etc.

* Se utilizaron en la etapa de difusión del proyecto.

\section{Equipamientos:}

Herramientas digitales educativas (Classmate, laptop $\mathrm{XO}$ ), equipos móviles, reproductores de medios digitales y las propias computadoras, que hoy en día son herramientas básicas dentro del proceso educativo.

\section{LOGROS RESULTANTES}

Logro 1: Mejora de lectura y escritura en las alumnas de la institución educativa, haciendo uso de las TIC.

- Disminución de dificultades en el proceso de lectoescritura.

- Aplicación de prácticas sociales apoyadas por las TIC, en el mejoramiento del proceso de la lectoescritura.

- Mejora de la comprensión de lectura en las alumnas del plantel.

\section{Logro 2: Protección el medio ambiente}

- Alumnas sensibilizadas en el recojo de basura.

- Disminución de conductas dañinas contra el medio ambiente.

- Difusión de los logros ambientales usando el blog del proyecto y las redes sociales. La comunicación intra familiar se incrementó, porque se involucra a los padres de familia en el cuidado del medio ambiente.

Logro 3: Fomento de la creatividad y expresión artística

- Alumnas sensibilizadas acerca de la importancia del arte en la educación y el desarrollo de la creatividad.

- Las estudiantes mejoran su auto percepción y opinión de sí mismas, al expresarse artísticamente.

- Incremento del trabajo en equipo en un contexto de armonía y colaboración.

\section{Lecciones aprendidas}

$1^{\circ}$ Mejorar nuestro proceso de lectoescritura desarrollando prácticas sociales, apoyadas por las TIC.

$2^{\circ}$ Confeccionar libros cartoneros, y poner en práctica nuestras habilidades personales y gustos propios.

$3^{\circ}$ Cuidar del medio ambiente y la biodiversidad, reutilizando el cartón. Y lo más relevante afianzar nuestra conciencia ambiental, que luego es reflejada en nuestros hogares.

$4^{\circ}$ Actuar con plena conciencia de nuestros derechos y deberes ambientales, además de brindar alternativas de solución a los problemas ambientales, 
específicamente de la región.

$5^{\circ}$ Incentivar la creatividad, permitiendo que nuestra imaginación se desarrolle al diseñar las tapas, y al crear textos para escribirlos en los libros cartoneros como: poesías, cuentos, acrósticos, pensamientos, adivinanzas, etc, de autoría propia, auxiliados por las TIC.

$6^{\circ}$ Interesarnos más en las áreas de Ciencia y Ambiente y Tecnología, además de aplicar el método científico para la investigación.

Finalmente, el proyecto que se ejecutó cumplió el objetivo principal, es decir, que se comprobó que las personas: ya sean adultos o niños, cuando confeccionan con sus propias manos libros de cartón, se sienten felices de poder reciclar y convertir los clásicos cartones de artefactos eléctricos, juguetes, leche, panetón, pizza, etc.; en libros originales y únicos. Así de alguna manera contribuir con el recojo de la basura y el cuidado del medio ambiente. Además se descubrió que de una manera menos ceremoniosa se da inicio al acto de la lectura y escritura, apoyados por las TIC, lo cual mejora el aprendizaje integral de las estudiantes.

\section{Webgrafía (Evidencias del proyecto):}

- Blog del Proyecto Cartonero: http://monicalanchipa.blogspot.com/

- Testimonios (docentes, padres de familia, estudiantes):

https://www.youtube.com/watch?v=pU

\section{Fotografías o imágenes de la ejecución del proyecto:}

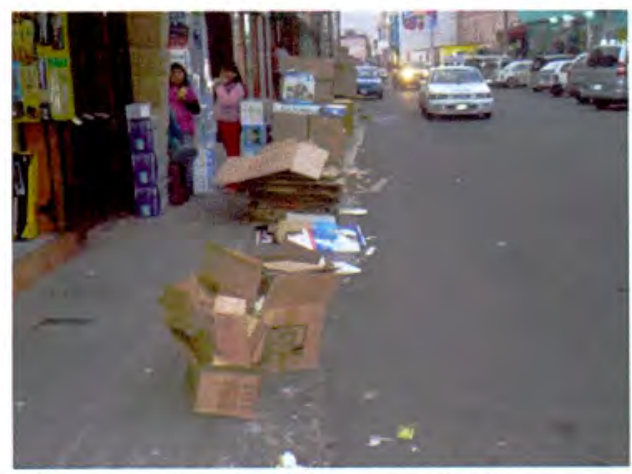

Figura 1. Recojo del cartón de los mercadillos de Tacna.

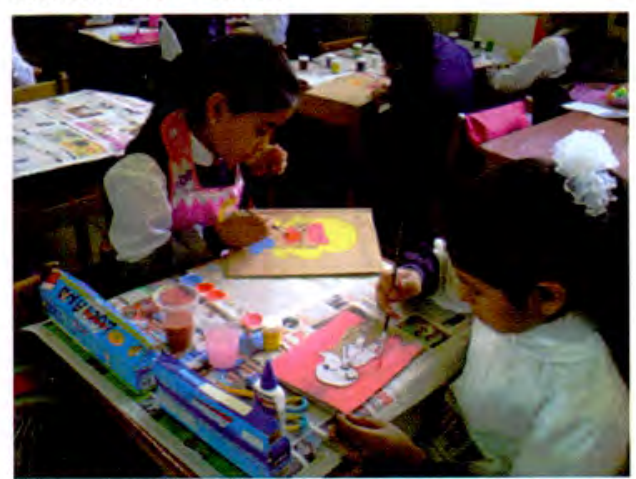

Figura 2. Elección del cartón y corte de las tapas.

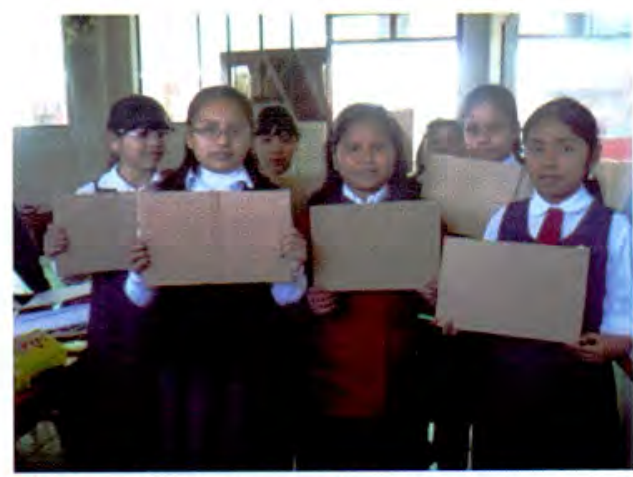

Figura 3. Diseño y pintado de las tapas del librito cartonero.

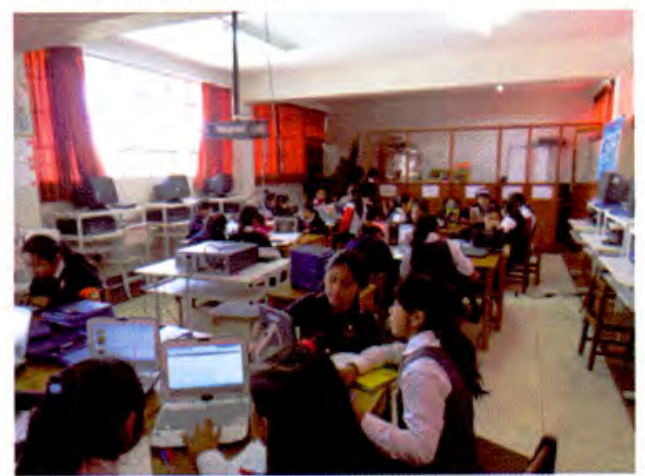

Figura 4. Usando herramientas digitales educativas (Classmate) para investigar, previo a las creaciones. 


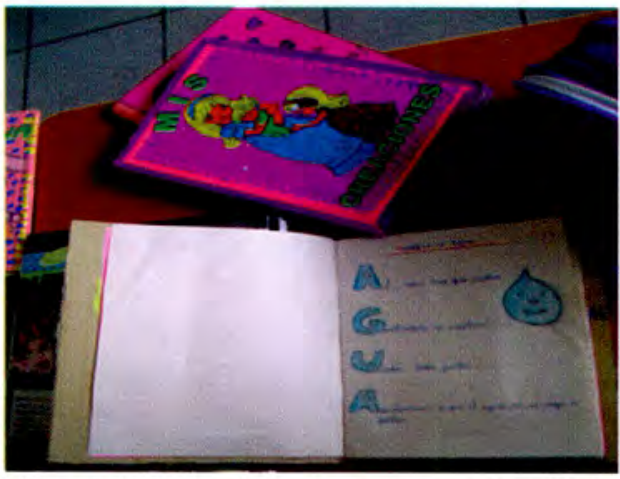

Figura 5. Creación de textos en los libritos cartoneros.

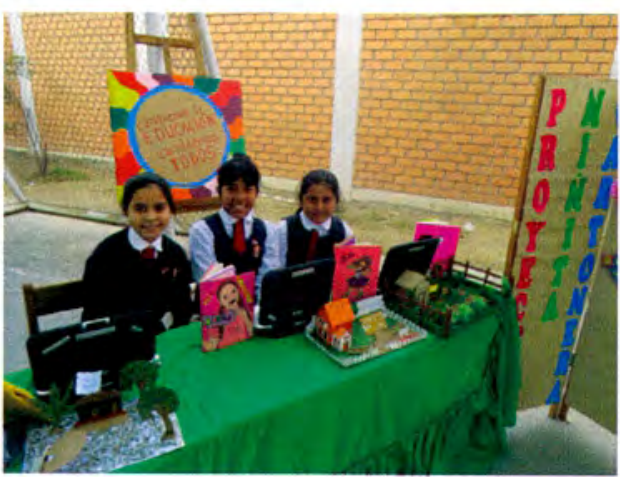

Figura 6. Los textos creados fueron compartidos con sus pares.

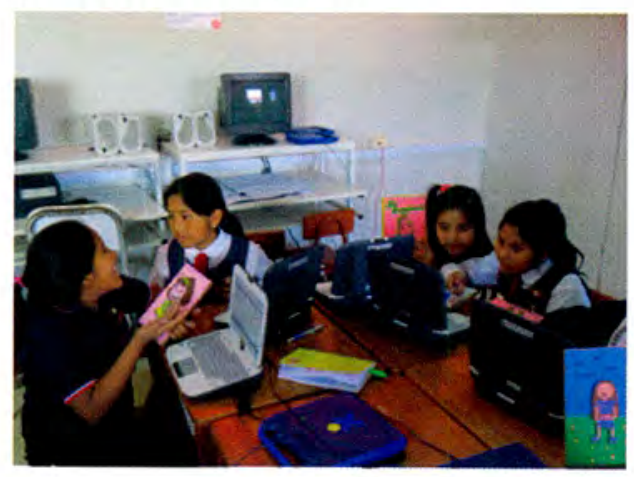

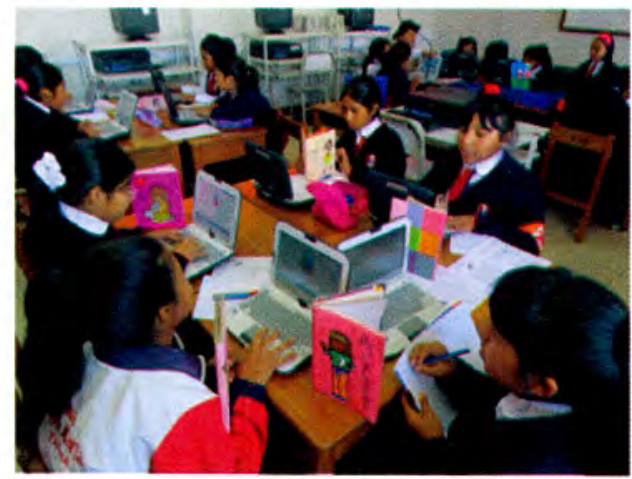

Figura 7. Las creaciones se difunden a través del blog y redes sociales del proyecto.

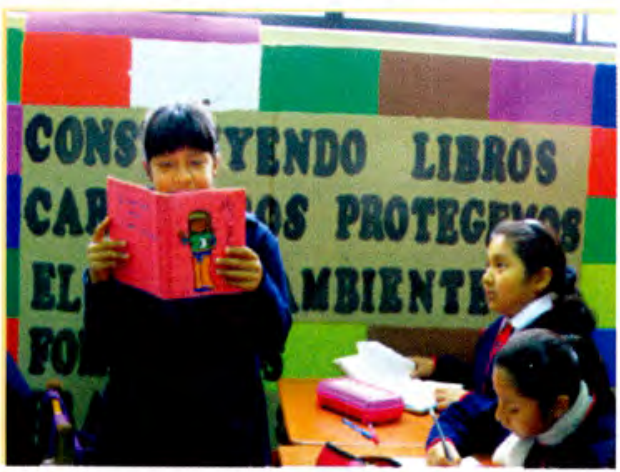

Figura 8. Se realizaron aprendizajes colaborativos con las compañeras de aula.

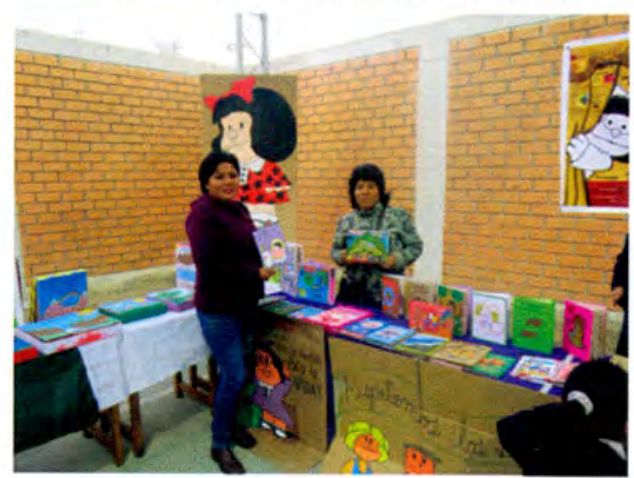

Figura 9. El proyecto fue difundido en los "Días de Logro"

Mcvj5TaKA

- Video del Proyecto "Construyendo Libros Cartoneros Protegemos el Medio Ambiente" Testimonios de la Familia Escolar 2014:

https://www.youtube.com/watch?v=pU Mcoj5TaKA
- Video del Proyecto Cartonero, con fondo musical, canción ecológica: "El Tiempo es hoy":

https://www.youtube.com/watch?v=k7 050IcqkHQ

- Video del I Encuentro Binacional de Integración Medio Ambiental, can- 
ción "Vientos de Amistad" de la I.E. Santísima Niña María:

https://www.youtube.com/watch?v=sU iSqXVkumo

- Video de la explicación de la construcción de un Librito Cartonero 2011 - I.E. Santísima Niña María de Tacna: https://www.youtube.com/watch?v=siSEi_o6iQElist $=H L 1403229628 \mathcal{E}$ index $=1$

- Video de ¿Cómo hacer un librito cartonero? 2013:

https://www.youtube.com/watch?v $=2 \mathrm{~V}$ js5YZlijc

- Presentación del proyecto a través de los años 2009-2013 y mensajes a la lectura:

http://www.docstoc.com/docs $/ 17128348$ 4/Proyecto-a-trav \% C3\% A9s-de-los$a \%$ C3\% B1os-2009-2013-y-mensajes- $a$ la-lecturappt

- Presentación del proyecto cartonero utilizando las TIC:

http://es.slideshare.net/lanbermo/pptde-proyecto-cartonero-mnica-tacna-per

- Presentación del proyecto cartonero 2009-2014 de la I.E. Santísima Niña María de Tacna-Perú:

http://es.slideshare.net/lanbermo/proyec to-cartonero-20092014-de-la-iesantsima-nia-mara-de-tacnaper? related $=1$

\section{CONCLUSIÓN}

Mediante la aplicación de las concepciones de reciclaje y aprendizaje colaborativo se logró de manera reconocida y exitosa el uso adecuado y útil de los residuos sólidos como el cartón en bene- ficio del medio ambiente.

\section{REFERENCIAS BIBLIOGRÁFICAS:}

Bartolome, A. (2015). Nuevas tecnologias en el aula. Guía de supervivencia. Madrid, España: Editorial Grao

Carneiro, R., Toscano, J. C. y Díaz, T. (s/f). Los desafios de las TIC para el cambio educativo. Madrid, España: Fundación Santillana. OEI

Carrió Pastor, M. L. (2007). Ventajas del uso de la tecnología en el aprendizaje colaborativo. Revista Iberoamericana de Educación, pp. 1-10.

Cassany, D. (2006). Taller de textos. Leer, escribir y comentar en el aula. España: Editorial Paidós.

Collazos, C., Guerrero, L. \& Vergara, A. (2001). Aprendizaje Colaborativo: un cambio en el rol del profesor. Proceedings of the 3rd Workshop on Education on Computing, Punta Arenas, Chile.

D'Angelo, E. \& Oliva, J. (2003). Lectura y escritura en contextos de diversidad. Madrid, España: D.G. Promoción Educativa.

Del VaL, A. (2011). El libro del reciclaje. Barcelona, España: RBA Libros

Suzuki, D y Vanderlinden, K. (2005), Tú eres la Tierra: Mejora el planeta del que formas parte. Barcelona, España: Editorial Oniro.

Tudge, C. (1994). Vigotsky: la zona de desarrollo próximo y su colaboración en la práctica de aula. Nueva York, Universidad de Cambridge. 